\title{
Non-local separable solutions of two interacting particles in a harmonic trap
}

\author{
C. González-Santander and F Domínguez-Adame \\ GISC. Departamento de Física de Materiales, Universidad Complutense, E-28040 \\ Madrid, Spain
}

\begin{abstract}
We calculate the energy levels of two particles trapped in a harmonic potential. The actual two-body potential, assumed to be spherically symmetric, is replaced by a projective operator (non-local separable potential) to determine the energy levels in a closed form. This approach overcomes the limitations of the regularized Fermi pseudopotential when the characteristic length of the two-body interaction potential is of the order of the size of the harmonic trap. In addition, we recover the results obtained with the Fermi pseudopotential when the length of the interaction is much smaller than the size of the trap.
\end{abstract}

Keywords: Harmonic confinement, two-interacting particles, non-local potential approach

PACS: 03.65.Nk 31.15.-p 34.50.Cx

\section{Introduction}

The rapid development of experimental techniques in the field of ultracold atoms has led to a renewed interest in the two-body problem, especially if the interacting atoms are subjected also to confinement potentials. In this context, Stöferle et al. studied two trapped ${ }^{40} \mathrm{~K}$ atoms in an optical lattice. They tuned the atom-atom interaction potential using a Feshbach resonance to form diatomic molecules from fermionic atoms [1]. The diatomic molecules were created in a deep optical lattice, when the tunneling between individual wells is suppressed and the three-dimensional (3D) confinement potential can be regarded as a harmonic trap. 
The problem of two cold atoms in a 3D harmonic trap was earlier considered theoretically in Ref. [2]. In this work, the actual atom-atom interaction potential was replaced by a zero-range potential [3], known as regularized Fermi pseudopotential. However, as the size of the harmonic trap decreases, it is apparent that the details of the interaction potential between atoms become more important. Therefore, atomic collisions in narrow harmonic traps are not expected to be accurately described by a zero-range potential. The lack of accuracy will appear when the range of the atom-atom interaction potential is comparable to the size of the harmonic trap.

The aim of this work is to develop a suitable approximation of the interaction potential to describe two-body collisions under strong confinement conditions. This study has two main goals. In the first place, we look for a replacement of the actual interaction potential by a non-local potential, while keeping the computational effort to a minimum. In other terms, we introduce a solvable model of the two-body problem in a 3D harmonic trap that allows us to obtain the energy levels in a closed form. In the second place, the results obtained with the regularized Fermi pseudopotential should be recovered when the size of the harmonic trap is much larger than the range of the two-body potential.

\section{The zero-range pseudopotential}

Consider two particles of mass $M$ trapped in a 3D harmonic potential of characteristic frequency $\omega$. After separating off the center-of-mass degree of freedom, the Hamiltonian for the relative motion is

$$
\mathcal{H}_{\mathrm{rel}}=-\frac{\hbar^{2}}{2 \mu} \nabla^{2}+\frac{1}{2} \mu \omega^{2} r^{2}+V(r) \equiv \mathcal{H}_{0}+V(r)
$$

where $\mu=M / 2$ is the reduced mass, $\boldsymbol{r}=\boldsymbol{r}_{1}-\boldsymbol{r}_{2}$ is the relative coordinate and $V(r)$ is the interaction potential, assumed to be spherically symmetric hereafter. The actual interaction potential is often replaced by a regularized Fermi pseudopotential of the form $[2,3,4,5]$

$$
V(r) \psi(\boldsymbol{r}) \longrightarrow \frac{\hbar^{2}}{2 \mu} 4 \pi a_{s} \delta(\boldsymbol{r}) \frac{\partial}{\partial r}[r \psi(\boldsymbol{r})]
$$

where $a_{s}$ is the $s$-wave scattering length. 
The interaction energy $E$ of the two particles can be calculated using the Green function for the 3D harmonic oscillator

$$
\left(\mathcal{H}_{0}-E\right) G\left(\boldsymbol{r}, \boldsymbol{r}^{\prime} ; E\right)=\delta\left(\boldsymbol{r}-\boldsymbol{r}^{\prime}\right)
$$

from which the corresponding eigenfunction is obtained as follows

$$
\psi(\boldsymbol{r})=-4 \pi a_{s} G(\boldsymbol{r}, 0 ; E) \frac{\partial}{\partial r^{\prime}}\left[r^{\prime} \psi\left(\boldsymbol{r}^{\prime}\right)\right]_{\boldsymbol{r}^{\prime}=0}
$$

The consistency of Eq. (4) leads to an implicit equation for the interaction energy

$$
1=-4 \pi a_{s} \frac{\hbar^{2}}{2 \mu} \frac{\partial}{\partial r}[r G(\boldsymbol{r}, 0 ; E)]_{\boldsymbol{r}=0} .
$$

The Green function associated to the 3D harmonic oscillator is well known [6]

$$
\begin{aligned}
G\left(\boldsymbol{r}, \boldsymbol{r}^{\prime} ; E\right) & =-\frac{1}{\hbar \omega}\left(\frac{\mu \omega}{\pi \hbar}\right)^{3 / 2} \exp \left(-\frac{r^{2}+r^{\prime 2}}{2 L^{2}}\right) \int_{0}^{1} z^{1 / 2-\epsilon}\left(1-z^{2}\right)^{-3 / 2} \\
& \times \exp \left(\frac{2 \boldsymbol{r} \cdot \boldsymbol{r}^{\prime} z-\left(r^{2}+r^{\prime 2}\right) z^{2}}{L^{2}\left(1-z^{2}\right)}\right) d z
\end{aligned}
$$

where $L=\sqrt{\hbar / \mu \omega}$ is the size of the harmonic trap and $\epsilon=E / \hbar \omega$. Inserting (6) in (5) and after a lengthly but straightforward algebra one gets

$$
\frac{L}{a_{s}}=2 \frac{\Gamma(3 / 4-\epsilon / 2)}{\Gamma(1 / 4-\epsilon / 2)},
$$

$\Gamma(z)$ being the Gamma function. This result is in agreement to that obtained earlier in Ref. [2].

\section{Finite-range interactions}

One way to obtain a solvable model when the two particles are subjected to a finite-range interaction is to start with a non-local separable potential (NLSP), which could be a reasonable approximation to the local potential. In this case the resulting equation can be exactly solved without tedious and elaborated calculations. What is more important, it is always possible to find an NLSP (or a sum of them) able to reproduce any set of given quantum states [7] and, consequently, there is no theoretical limitation to the numerical 
accuracy with which physical results can be obtained. NLSPs have proved useful in the study of few-body problems in nuclear and condensed matter physics $[8,9,10,11,12]$.

The NLSP method replaces $V(r)$ in (1) by a projective operator of the form

$$
V(r) \psi(\boldsymbol{r}) \rightarrow-\frac{\lambda \hbar^{2}}{2 \mu} u(\boldsymbol{r}) \int d^{3} \boldsymbol{r}^{\prime} u\left(\boldsymbol{r}^{\prime}\right) \psi\left(\boldsymbol{r}^{\prime}\right)
$$

where we set $\lambda= \pm 1$ hereafter without loss of generality, namely the strength of the potential is embodied in the shape function $u(\boldsymbol{r})$.

The Schrödinger equation for the potential (8) reduces to an algebraic equation in momentum space (see, e.g., Ref. [13]). Therefore, the NLSP method allows all magnitudes of interest, such as resonance energy, phase shift or bound state energy, to be expressed in a closed form. In particular, the $s$-wave scattering phase shift $\delta_{0}(\boldsymbol{k})$ satisfies the equation [9]

$$
k \cot \delta_{0}(\boldsymbol{k})=\frac{1}{4 \pi \lambda|\widetilde{u}(\boldsymbol{k})|^{2}}\left[1+\frac{2 \lambda}{\pi} \mathcal{P} \int d^{3} \boldsymbol{q} \frac{|\widetilde{u}(\boldsymbol{q})|^{2}}{k^{2}-q^{2}}\right],
$$

where $\mathcal{P}$ denotes the principal value and

$$
\widetilde{u}(\boldsymbol{k})=\frac{1}{4 \pi} \int d^{3} \boldsymbol{r} e^{i \boldsymbol{k} \cdot \boldsymbol{r}} u(\boldsymbol{r})
$$

is the Fourier transform of the shape function.

\subsection{Yamaguchi NLSP}

The Yamaguchi NLSP, $u(r)=(g / r) \exp (-r / a)$, was introduced to describe nucleon-nucleon interaction in nuclear physics [14]. Later, it was also applied to describe Wannier excitons in quantum dots [15]. The scattering length and the effective range of the potential depends on the parameter $a$. From Eq. (9) we get

$$
k \cot \delta_{0}(k)=-\frac{1}{2 a}+\frac{\left(1+k^{2} a^{2}\right)^{2}}{\xi a},
$$

where $\xi=4 \pi \lambda g^{2} a^{3}$. Taking into account that at low energy $k \cot \delta_{0}(k) \simeq$ $-1 / a_{s}+(1 / 2) r_{0} k^{2}$, where $r_{0}$ is the effective range of the interaction potential, one gets

$$
a_{s}=a\left(\frac{1}{2}-\frac{1}{\xi}\right)^{-1}, \quad r_{0}=\frac{4 a}{\xi}
$$


When $\lambda$ is positive the NLSP supports a bound state if $\xi>2$, i.e. $a_{s}>0$. The unnormalized eigenfunction is

$$
\psi_{0}(\boldsymbol{r})= \begin{cases}\frac{1}{r}\left(e^{-k_{0} r}-e^{-r / a}\right), & k_{0} \neq a^{-1} \\ e^{-r / a}, & k_{0}=a^{-1}\end{cases}
$$

and the corresponding energy is $E_{0}=-\hbar^{2} k_{0}^{2} / 2 \mu$, where $k_{0}$ is given by

$$
k_{0}=\frac{1}{a}[\sqrt{\xi / 2}-1]^{1 / 2} .
$$

\section{2. $\delta$-shell $N L S P$}

As a second working example of NLSP we consider the $\delta$-shell, $u(r)=$ $g \delta(r-a)[15]$. In this case, from Eq. (9) we obtain

$$
k \cot \delta_{0}(\boldsymbol{k})=-k \cot k a+\frac{k^{2} a}{\xi \sin ^{2} k a},
$$

whose limit at low energy leads to

$$
a_{s}=a\left(1-\frac{1}{\xi}\right)^{-1}, \quad r_{0}=\frac{2}{3} a\left(1+\frac{1}{\xi}\right) .
$$

The $\delta$-shell NLSP supports a bound state when $\lambda$ is positive and $\xi>1$ $\left(a_{s}>0\right)$. In this case the unnormalized eigenfunction is

$$
\psi_{0}(\boldsymbol{r})=\frac{1}{r}\left[e^{-k_{0}|r-a|}-e^{-k_{0}(r+a)}\right],
$$

where $k_{0}$ is the root of the transcendent equation

$$
1=\frac{1-e^{-2 k_{0} a}}{2 k_{0} a} \xi,
$$

and the energy of the bound state is $E_{0}=-\hbar^{2} k_{0}^{2} / 2 \mu$.

\section{Two particles in a harmonic trap}

The collision properties of the two particles whose interaction is described by a NLSP are strongly modified when they are placed in a harmonic trap. 
The solution of the Schrödinger equation for the relative motion can be expressed as

$$
\psi(\boldsymbol{r})=-\frac{\hbar^{2} \lambda}{2 \mu} \int d^{3} \boldsymbol{r}^{\prime} G\left(\boldsymbol{r}, \boldsymbol{r}^{\prime} ; E\right) u\left(r^{\prime}\right) \mathcal{I}
$$

with

$$
\mathcal{I}=\int d^{3} \boldsymbol{r} u(r) \psi(\boldsymbol{r})
$$

where the Green function is given by (6). After inserting (19) into (20) and performing the angular integration we obtain the following equation for the energy of the relative motion

$$
\frac{\mu}{2 \pi \lambda \hbar^{2}}=-\iint d r d r^{\prime}\left(r r^{\prime}\right)^{2} \mathcal{G}\left(r, r^{\prime} ; E\right) u(r) u\left(r^{\prime}\right)
$$

where the radial Green function is given by [16]

$$
\begin{aligned}
\mathcal{G}\left(r, r^{\prime} ; E\right) & =\frac{\Gamma(3 / 4-\epsilon / 2)}{\Gamma(3 / 2) L^{3} \hbar \omega} \exp \left(-\frac{r^{2}+r^{\prime 2}}{2 L^{2}}\right) \\
& \times M\left(3 / 4-\epsilon / 2,3 / 2, r_{<}^{2} / L^{2}\right) U\left(3 / 4-\epsilon / 2,3 / 2, r_{>}^{2} / L^{2}\right)
\end{aligned}
$$

$M$ and $U$ being the confluent hypergeometric functions [17]. Here $r_{>}$and $r_{<}$ denotes the largest and the smallest value of the pair $\left(r, r^{\prime}\right)$, respectively.

\subsection{Yamaguchi NLSP}

When $u(r)$ is the Yamaguchi potential, the integration in (21) cannot be expressed in a closed form. However, it can be obtained easily by numerical methods since both the Green function and the Yamaguchi potential fall off rapidly with the distance. In addition, using the asymptotic limits [17]

$$
M(\alpha, 3 / 2, z) \simeq 1, \quad U(\alpha, 3 / 2, z) \simeq \frac{\sqrt{\pi / z}}{\Gamma(\alpha)}-\frac{2 \sqrt{\pi}}{\Gamma(\alpha-1 / 2)},
$$

when $z \rightarrow 0^{+}$we recover the result obtained with the zero-range pseudopotential (7) for $a=0$. Thus, we come to the conclusion that the low-energy scattering properties of the regularized pseudopotential (7) and those of the Yamaguchi NLSP when $a \rightarrow 0$ are the same.

Figure 1(a) depicts the dependence of the lowest energy level on the positive scattering length $a_{s}>0$ for different values of the parameter $a$, in the case of the Yamaguchi NSLP. Energy is measured in units of $\hbar \omega$ and 
length is expressed in units of $L$. We observe that the curves shift to the high scattering length side of the plot on increasing the value of $a / L$. A more detailed inspection of the numerical solution of equation (21) reveals that the lowest energy level increases smoothly as a function of the parameter $a$ when $a_{s} \rightarrow \infty$ (weakly interacting particles) and approaches the limiting value $E=(3 / 2) \hbar \omega$. This is shown in figure $1(\mathrm{~b})$, where we have taken $a_{s} / L=60$ to solve equation (21) numerically, but we have checked that the results remain unchanged within the numerical uncertainty when $a_{s} / L=70$.
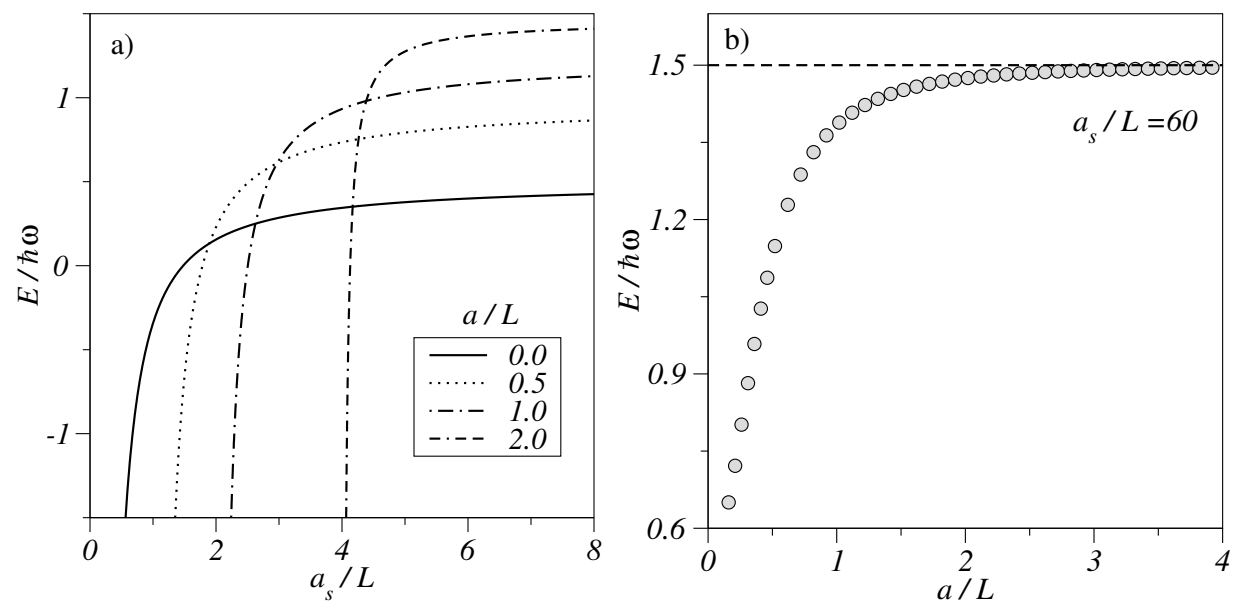

Figure 1: a) Lowest energy level in the case of the Yamaguchi NLSP, in units of $\hbar \omega$, as a function of the scattering length, expressed in units of $L$, for different values of the parameter $a$. b) Lowest energy level as a function of $a / L$ for $a_{s} / L=60$.

\section{2. $\delta$-shell NLSP}

In the case of the $\delta$-shell, we arrive at the following equation for the interaction energy from equation (21)

$$
\begin{aligned}
\frac{L}{a_{s}} & =\frac{L}{a}-\frac{\Gamma(3 / 4-\epsilon / 2)}{\sqrt{\pi}} \exp \left(-\frac{a^{2}}{L^{2}}\right) \\
& \times M\left(3 / 4-\epsilon / 2,3 / 2, a^{2} / L^{2}\right) U\left(3 / 4-\epsilon / 2,3 / 2, a^{2} / L^{2}\right) .
\end{aligned}
$$

Using the asymptotic limits given by (23) we recover again the result obtained with the zero-range pseudopotential (7).

Figure 2(a) shows the lowest energy level as a function of the positive scattering length for different values of the parameter $a$, in the case of the 
$\delta$-shell NSLP. The results resemble qualitatively those obtained with the Yamaguchi NLSP, shown in figure 1(a), including the energy decrease without bound as the scattering length vanishes (strongly interacting particles). In the opposite limit, when $a_{s} \rightarrow \infty$, the lowest energy level increases smoothly when the parameter $a$ increases, and approaches the limiting value $E=$ $(3 / 2) \hbar \omega$. This behaviour is shown in figure 2(b). Furthermore, using the asymptotic limits of the confluent hypergeometric functions [17], one can obtain the following expression for the lowest energy state when $a / L \gg 1$ and $a_{s} \rightarrow \infty$

$$
\frac{E}{\hbar \omega} \approx \frac{3}{2}-\frac{2 a}{\sqrt{\pi} L} \exp \left(-\frac{a^{2}}{L^{2}}\right)
$$

The solid line in the inset of figure 2(b) represents the energy obtained from equation (25), which is fully consistent with the numerical results for $a / L>3$.
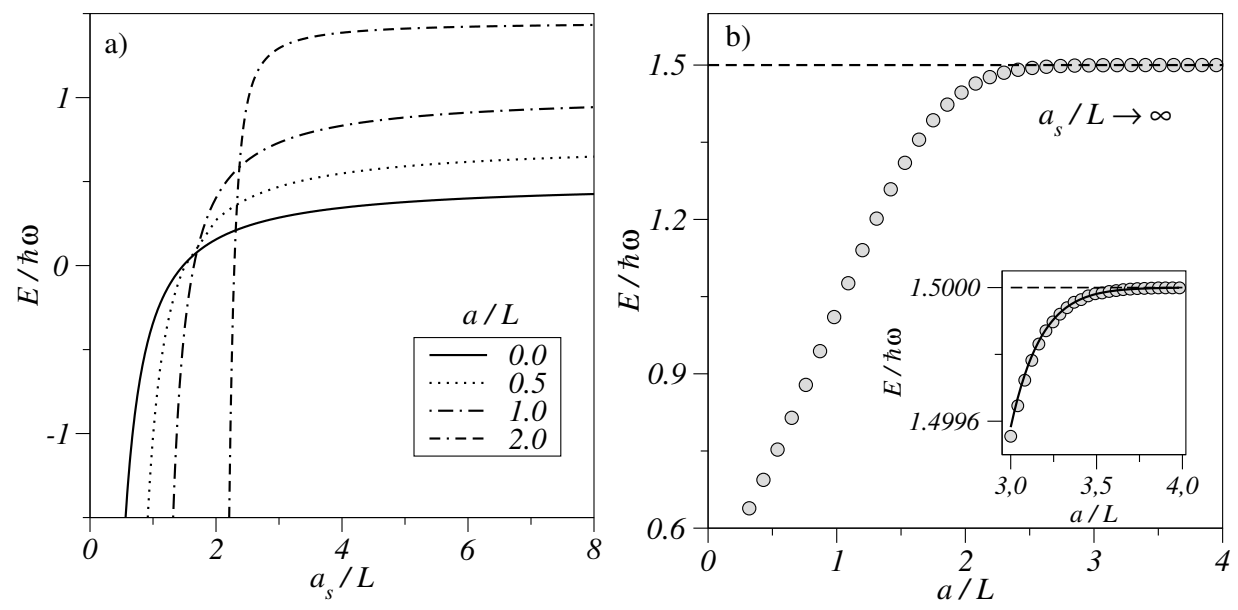

Figure 2: a) Lowest energy level in the case of the $\delta$-shell NLSP, in units of $\hbar \omega$, as a function of the scattering length, expressed in units of $L$, for different values of the parameter $a$. b) Lowest energy level as a function of $a / L$ when $a_{s} \rightarrow \infty$. The solid line in the inset corresponds to the energy obtained from equation (25).

Finally, figure 3 compares the first few levels of the zero-range pseudopotential and the NLSPs (Yamaguchi and $\delta$-shell), for positive and negative scattering length. It is important to stress that the results for the zero-range pseudopotential (dashed line of figure 3) can be recovered from the NLSP approach by taking $a \ll L$. If the parameter $a$ is of the order of the size of the harmonic trap, the NLSP predicts a remarkable increase of the energy levels 
(solid and dash-dotted lines of figure 3) as compared to the the zero-range pseudopotential result, when the magnitude of the $s$-wave scattering lengh $a_{s}$ is large (irrespective of its sign). Also notice that the interaction energy obtained with the NLSPs reaches an asymptotic limit when $a_{s}$ is negative or $a_{s}>2 L$.

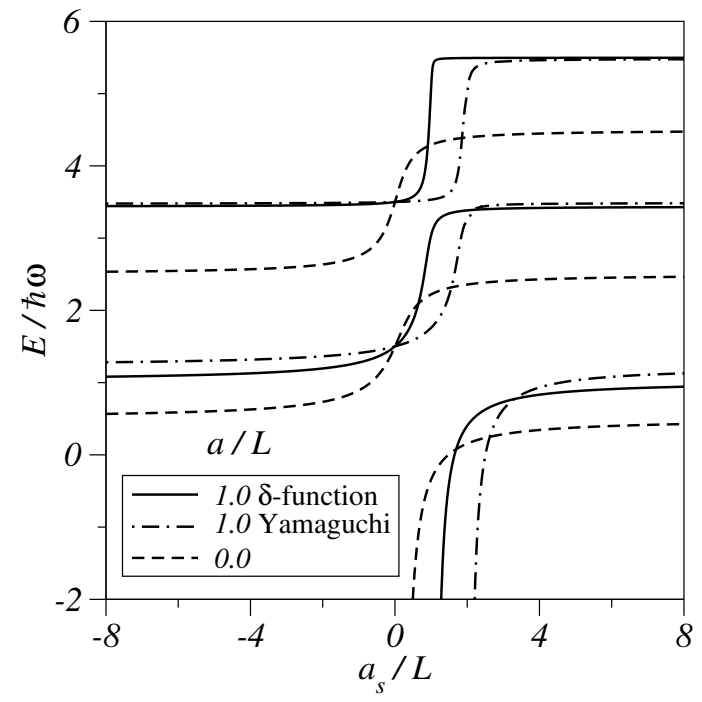

Figure 3: Energy levels of the two particles in a harmonic trap, in units of $\hbar \omega$, as a function of the scattering length, expressed in units of $L$, for different values of the parameter $a$. Results correspond to the zero-range pseudopotential and the NLSPs (Yamaguchi and $\delta$-shell), as indicated in the legends.

\section{Conclusions}

We have shown how one can construct a solvable model based on nonlocal separable potentials to calculate the energy levels of two particles trapped in a harmonic trap. The interaction energy can be obtained in a closed form by solving the transcendent equation (21), for any arbitrary nonlocal separable potential. As a working example, we considered the Yamaguchi and the $\delta$ shell NLSPs, and compared the predicted energy levels to those obtained by a regularized zero-range pseudopotential. In both cases, the low-energy scattering properties of the nonlocal potentials approach those shown by the regularized zero-range pseudopotential when the parameter $a$ vanishes.

The authors thank J. M. R. Parrondo for discussions. This work was supported by MICINN (projects Mosaico and MAT2010-17180). C. G.-S. 
acknowledges financial support from Comunidad de Madrid and European Social Foundation.

[1] T. Stöferle, H. Moritz, K. Günter, M. Köhl, and T. Esslinger, Phys. Rev. Lett. 96, 030401 (2006).

[2] T. Busch, B.-G. Englert, K. Rzążewski, and M. Wilkens, Found. Physics 28, 549 (1998).

[3] Yu. N. Demkov and V. N. Ostrovskii, Zero-Range Potentials and their Applications in Atomic Physics (Plenum Press, New York, 1998).

[4] E. Tiesinga, C. J. Williams, F. H. Mies, and P. S. Julienne, Phys. Rev. A 61, 063416 (2000).

[5] M. Köhl, K. Günter, T. Stöferle, H. Moritz, and T. Esslinger, J. Phys. B: At. Mol. Opt. Phys. 39, S47 (2006).

[6] E. Capelas de Oliveira, Rev. Bra. Fis. 3, 697 (1979).

[7] M. L. Glasser, Surf. Sci. 64, 141 (1977)

[8] W. van Dijk, Phys. Rev. C 40, 1437 (1989).

[9] T. Gherghetta and Y. Nambu, Int. J. Mod. Phys. 8, 3163 (1993)

[10] F. Domínguez-Adame, E. Diez, and A. Sánchez, Phys. Rev. B 51, 8115 (1995).

[11] W. van Dijk, M. W. Kermode, and S. A. Moskkowski, J. Phys. A: Math. Gen. 31, 9571 (1998).

[12] C. González-Santander and F. Domínguez-Adame, Physica E 41, 1645 (2009).

[13] B. W. Knight and G. A. Peterson, Phys. Rev. 132, 1085 (1963).

[14] Y. Yamaguchi, Phys. Rev. 95, 1628 (1954).

[15] S. López and F. Domínguez-Adame, Semicond. Sci. Technol. 17, 227 (2002).

[16] V. L. Bakhrakh and S. I. Vetchinkin, Theor. Math. Phys. 6, 283 (1971). 
[17] M. Abramowitz and I. Stegun, Handbook of Mathematical Functions (Dover, New York, 1972). 